\title{
Influence of ion implantation and annealing on composition and structure of GaAs surface
}

\author{
M.T. Normuradov ${ }^{1)}$, B.E. Umirzakov²), D.A. Tashmukhamedova ${ }^{2)}$, A.K. Tashatov ${ }^{1)}$ \\ ${ }^{1)}$ Karshin Engineer-and-economics \\ ${ }^{2)}$ Tashkent State Technical University \\ E-mail:ftmet@rambler.ru
}

\begin{abstract}
In this work investigated is the influence of barium ion implantation and subsequent annealing on composition, electronic and crystalline structure of GaAs surface. For the first time, the influence of low energy $\mathrm{Ba}^{+}$ions implantation on the structure of GaAs surface was investigated using photoelectron spectroscopy. Determined are parameters of energy bands and the crystalline lattice of the three-component system $\mathrm{Ga}_{0.6} \mathrm{Ba}_{0.4}$ As. The width of the forbidden gap is $E \mathrm{~g}=1 \mathrm{eV}$, the lattice constant is $a=5.73 \AA$.
\end{abstract}

Keywords: ion-implantation, photoelectron spectroscopy, post-implantation annealing.

Paper received 29.03.01; revised manuscript received 03.06.02; accepted for publication 25.06.02.

\section{Introduction}

From the viewpoint of creation of solar cells, integrated circuites, optoelectronic and integrated-optical systems, it is very important to obtain new multi-component thinfilm structures grown on surfaces of each other. In particular, criteria of applicability of solar cells in cosmic energetics are as follows: high efficiency of solar energy transformation and stability to radioactive emission. In this respect, special interest presents creation of periodic and layered systems with variable composition based on GaAs films.

One of the efficient methods to obtain these structures is the low energy ion implantation. Many works [1-4] were aimed to study the influence of ion implantation on condition and properties of solid surface. These investigations showed that low energy ion bombardment, results in improvement of emission efficiency of material, promotes low-temperature epitaxial growth of films from ion-molecular beams, allows to get passivating, anticorrosive and other coatings $[3,4,8]$. There were also obtained reliable data about formation of the chemical bonds between atoms of matrixes and an implanted element, about degrees of surfaces disorder, about changes of positions intrinsic to maxima of valent electron density states $[3,4,9]$. However, taking into account the defi- ciency of the systematic researches using the most informative methods of surface diagnostics, a whole series of questions, related to changes of the electronic states, atomic structure and mechanisms of new phase formation were not ascertained so far. In particular, there was practically absent reliable information about the form and structure of the electronic density-of-state spectrum and about the nature of features observed in these spectra for crystals, implanted by low energy ions $\left(E_{0} \leq 10 \mathrm{keV}\right)$ and subjected to the following annealing.

In this work, investigated was the effect of barium ions implantation and of the subsequent annealing on composition, electronic and crystalline structures of $\mathrm{GaAs}$ surface.

\section{Experimental}

The technological treatment (ion-implantation, annealing), the investigation of the structure and properties of samples were carried out in the same high vacuum $\left(P \leq 10^{-7} \mathrm{~Pa}\right)$ experimental device. The experimental device and measurement procedure were described in details earlier [4]. A change of the surface crystalline structure were studied by high-energy electron diffraction (HEED) using a standard device of the EMR-2 type. 


\section{M.T. Normuradov et al.: Influence of ion implantation and annealing...}

For carrying out implantation of barium ions, used was the construction of gun based on the principle of surface ionization [5]. BaTi tablets were used as an ion source. The ion guns contain the following main parts: ion source, accelerating and focusing electrodes, separator of ions on masses. The ion gun formed a monoenergetic ion beam with an energy $E_{0}$ between 0.5 and $5 \mathrm{keV}$ and a current density $j$ in the range 0.5 $20 \mu \mathrm{A} \mathrm{cm}{ }^{-2}$. The diameter of the ion beam at the target was $4 \mathrm{~mm}$.

For determination of atoms matrixes and implanted element concentration profiles used was the Auger - electron spectroscopy (AES) method in combination with ionic etching. The etching was carried out using $\mathrm{Ar}^{+}$ions with $E_{0}=3 \mathrm{keV}$ at an angle of $\sim 3^{0}$ relatively to surfaces.

As an object of investigation we used the face (110) of a GaAs monocrystal. Before ion-implantation, a surface GaAs was cleaned by the high-temperature annealing in combination with soft laser irradiation.

\section{Results}

Implantation of $\mathrm{Ba}^{+}$ions was carried out under fixed energy $\left(E_{0}=\right.$ const $)$ ranged within 0.5 to $5 \mathrm{keV}$. In doing so, the most important change of the state and properties of GaAs surface layers occured at relatively low energies of ions. So, in this work, the main results are related to $E_{0}=0.5 \mathrm{keV}$.

The photoelectron spectra for a fixed photon energy of $h v=10.8 \mathrm{eV}$ for GaAs (110) implanted by barium ions having energy $E_{0}=0.5 \mathrm{keV}$ with different doses shown in Fig. 1. On the basis of analysis of photoelectron spectra, it is possible to get information on distribution of density of electronic states and parameters of energy bands [6].

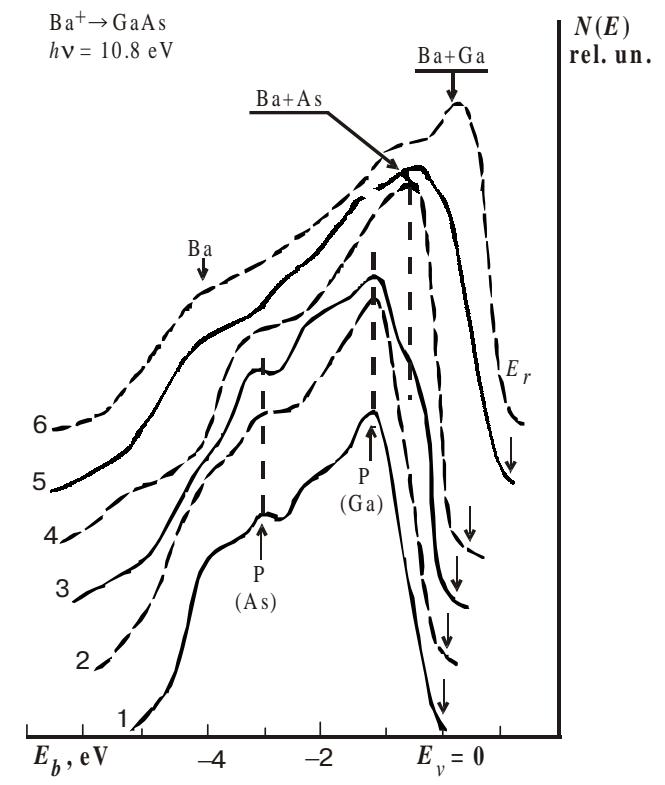

Fig. 1. Photoelectron spectra for p-type GaAs (110), implanted with $E_{0}=0.5 \mathrm{keV} \mathrm{Ba}^{+}$ions and doses $\mathrm{D}, \mathrm{cm}^{-2}: 1-0 ; 2-2 \cdot 10^{14} ; 3$ $-8 \cdot 10^{14} ; 4-6 \cdot 10^{15} ; 5-2 \cdot 10^{16} ; 6-8 \cdot 10^{16}$.
The energy bond $E_{\mathrm{eb}}$ of electrons is axis of abscissas. For all photoelectron energy distribution curves (PED) we used the same scale in vertical, chosen in such manner, that the area under each curve proportional to the value an electron quantum yield from samples. It is seen that all curves have a thin structure. In case of unimplanted GaAs, formation of the thin PED structure is mainly contributed by $\mathrm{p}$ - orbitals of Ga and As, as well as As s-states [7]. Observe that peak Ga with $E_{e b}=-1 \mathrm{eV}$ and peak As with $E_{e b}=-3 \mathrm{eV}$ is typical only for bound states. Initial part of the spectrum contains some subsidiary features, evidently, connected with surface states (curve 1). Ionimplantation depending on dose of irradiation results in a change of the photoelectron spectrum structure. Already at the dose $D=8 \cdot 10^{14} \mathrm{~cm}^{-2}$, there arises a new feature with the energy bond $\mathrm{E}_{\mathrm{eb}}=-0.6 \mathrm{eV}$ in the spectrum. In the range $D=8 \cdot 10^{14}-6 \cdot 10^{15} \mathrm{~cm}^{-2}$, intensity of this feature quickly increases with the growing dose. At that intensity of $\operatorname{GaAs}\left(E_{e b}=-1 \mathrm{eV}\right)$ main peak, appreciably decreases which is caused by excitation of $\mathrm{Ga} 4 \mathrm{p}$ electrons, and the As peak with $E_{e b}=-3 \mathrm{eV}$ noticeably widens slightly increasing in amplitude. The analysis of the data obtained by ultra-violet photoelectron spectroscopy (UVPS) together with the data of AES have shown that within this range of irradiation doses the process of ion implantation is followed by disordering the surface layer, decomposition of GaAs by components, some enriching of the surface with unbound As atoms at the expense of their prior diffusion to surfaces. Evidently, these As atoms at once enter into the chemical bond with interstitial $\mathrm{Ba}$ atoms, and separation of a certain quantity of atomic gallium takes place. Therefore, appearance of new peak in spectrum of photoelectrons is explained by formation of $\mathrm{Ba}+\mathrm{As}$ compounds, and the displacement of Fermi level $\left(E_{\mathrm{F}}\right)$ position by initiation of deep levels in the forbidden semiconductor gap, as a consequence of separation of atomic gallium, accompanied by formation the different defects in the crystalline structure. (In fact, $E_{\mathrm{F}}$ position is not changed, and displaced is the top edge of the valent band $E_{V}$ and the bottom of the conductivity band $E_{C}$, at the expense of formation of 0impurity levels near it).

At $D=(6-8) \cdot 10^{15} \mathrm{~cm}^{-2}$, ion-implanted layer of GaAs still keeps the properties typical of semiconductors (the width of the forbidden gap is $\sim 0.8 \mathrm{eV}$ ). For further growth of dose in near surface range the concentration of arsenic decreases (through partial desorption from surfaces and certain diffusion deep into the sample), while the near surface range is enriched by $\mathrm{Ba}$ atoms, part of which can form certain bonds with Ga atoms. Accordingly, their contribution to formation of photoelectrons increases. All this results in sharp changes of the structure of PED curves: the spectrum front is displaced by $\sim 1 \mathrm{eV}$ to the high energy side, and its form becomes typical for metalalloy emitters. These changes occur up to $D=8 \cdot 10^{16} \mathrm{~cm}^{-}$ 2 . Further increase of the irradiation dose doesn't result in noticeable changes of the dependence $N(E)$ (Fig. 1, curve 6). In this case, ion-implanted layers are completely disordered, and on the curve $N(E)$ one can observe, 
mainly, characteristics, intrinsic to the system $[\mathrm{Ba}+\mathrm{Ga}]$ and the clean barium, i.e. there occurs «metallization» of near surface GaAs layers. Thickness of these layers at $E=0.5 \mathrm{keV}$ are $25-30 \AA$.

Post-implantation high-temperature or laser annealing can modify the structure and properties of the surface

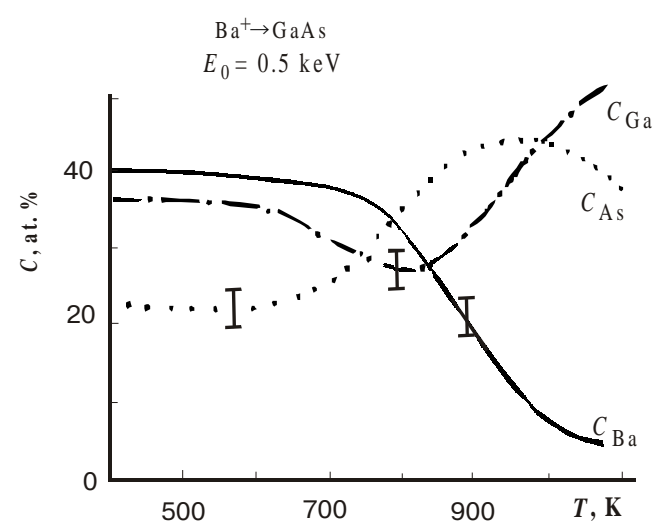

Fig. 2. Influence of the temperature on $\mathrm{Ba}, \mathrm{Ga}$ and $\mathrm{As}$ atoms surface concentration for GaAs implanted with $E_{0}=0.5 \mathrm{keV} \mathrm{Ba}{ }^{+}$ ions.

of the ion-implanted materials. Such annealing promotes the crystallization of disordered layers, formation of new compounds, decrease of the concentration of unbound substrate and implanted element atoms. In the Fig. 2 shown are the concentration profiles of $\mathrm{Ba}$, As and $\mathrm{Ga}$ versus temperature $T$, for GaAs implanted by $\mathrm{Ba}^{+}$with $E_{0}=0.5 \mathrm{keV}$. The samples were annealed for $30 \mathrm{~min}$. It is seen that main changes in concentrations of atoms occurs from $T=650-700 \mathrm{~K}$. With growing $T$, barium concentration monotonically decreases, the curve $C_{G a}(T)$ at $T=800 \mathrm{~K}$ passes across its minimum, and the curve $C_{A s}(T)$ at $T=900-950 \mathrm{~K}$ across its maximum. The analysis of the data of AES has shown that at $\mathrm{T} \geq 700 \mathrm{~K}$ practically all $\mathrm{Ba}$ atoms forms chemical bonds with matrix atoms. In the range $T=700-1000 \mathrm{~K}$, mainly formed are three-component compounds of the $\mathrm{Ga}_{1-\mathrm{x}} \mathrm{Ba}_{\mathrm{x}}$ As type, where $x$ value depends on $T$. For example, at $T=800 \mathrm{~K}$ value $x=0.5$, and at $T=900 \mathrm{~K}-x=0.4$. Further increase of the temperature results in decomposition of three-component compounds and intensive evaporation of their components from surfaces. Starting from $T=$ $700 \mathrm{~K}$, the change of the near surface layer composition is accompanied by recrystallization of disordered layers. In the range of $T=700-800 \mathrm{~K}$, there are concentric circles on HEED pictures, which is typical of polycrystalline films. There are point reflexes, inherent to singlecrystals in HEED pictures starting from 850 $900 \mathrm{~K}$. The type and parameters of this system lattice are determined. The three-component system had the cubic lattice, which is similar to that of substrate, i.e. the epitaxy film was formed. The parameter of this lattice was $a=$ $5.73 \pm 0.03 \AA$ which is some longer than $a$ for $\mathrm{GaAs}$ $(\sim 5.65 \AA)$.

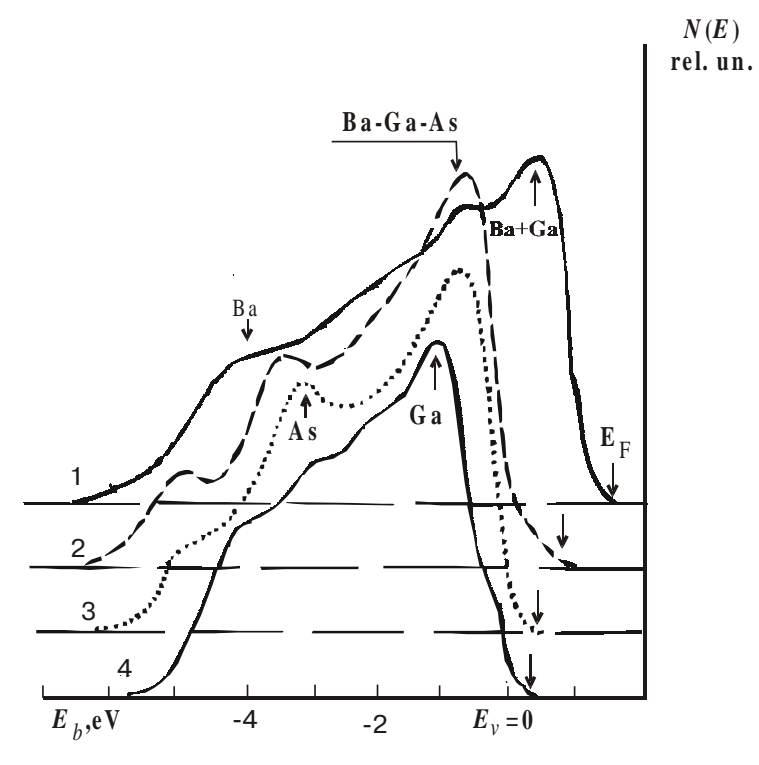

Fig. 3. Influence of temperature annealing on photoelectron spectra of GaAs implanted with $E_{0}=0.5 \mathrm{keV} \mathrm{Ba}^{+}$ions under $T, \mathrm{~K}: 1$ - 300; 2 - 800; 3 - 900; $4-1000$.

The dynamics of changing the PED curve for different $T$ in GaAs implanted with $E_{0}=0.5 \mathrm{keV} \mathrm{Ba}^{+}$ions is shown in Fig. 3. It is seen that at $T=800 \mathrm{~K}$ peaks with $E_{e b}=0.6$ and $-4.2 \mathrm{eV}$ typical to alloys $\mathrm{Ba}-\mathrm{Ga}$ of disappear, and new features appear. Evidently, new peaks appear in consequence of forming the three-component compound of $\mathrm{Ba}-\mathrm{Ga}-$ As type. Under these conditions, semiconductor properties of near surface layer becomes restore. The width of this system forbidden gap is $0.6 \mathrm{eV}$. With $T$ growing up to $900 \mathrm{~K}$, intensity of peaks with energy bonds of -0.7 and $-5 \mathrm{eV}$ sharply decreases, and intensity of peak $-3.2 \mathrm{eV}$ increases only a little (Fig. 3, curve 3 ). These changes are probably caused by decreasing content of barium in the system [Ba-Ga -As] and by growth of arsenic concentration in it. At $T=900 \mathrm{~K}$ the compound $\mathrm{Ga}_{0.6} \mathrm{Ba}_{0.4} \mathrm{As}$ is formed. For this system the width of forbidden gap is equal to $\sim 1.0 \mathrm{eV}$, i.e. these films are heterostructural. All features typical of GaAs, in the photoelectron spectrum, are fixed only after long annealing ( 3 hours and more) of sample at $T=1000-$ $1100 \mathrm{~K}$.

\section{Conclusions}

For the first time, researched is the influence of low energy $\mathrm{Ba}^{+}$ions implantation on the structure of photoelectron spectra of GaAs surfaces. It is determined that implantation of $\mathrm{Ba}^{+}$ions results in the formation of compounds of the BaAs type at doses of $D=8 \cdot 10^{14}$ $6 \cdot 10^{15} \mathrm{~cm}^{-2}$, and intermetallic $\mathrm{Ga}+\mathrm{Ba}$ compounds at doses $D \geq 10^{16} \mathrm{~cm}^{-2}$.

Post implantation annealing starting with $T=700-$ $750 \mathrm{~K}$ results in recrystallization of amorphous layers 


\section{M.T. Normuradov et al.: Influence of ion implantation and annealing...}

and redistribution of the atoms of matrixes and implanted ions in ion-implanted layer. At $T=900 \mathrm{~K}$, there occurs the full crystallization of the near surface layer and formation of the epitaxial $\mathrm{Ga}_{0.6} \mathrm{Ba}_{0.4} \mathrm{As}$ film with the thickness $30-40 \AA$.

The determined parameters of energy bands and crystalline lattice in the three-component system $\mathrm{Ga}_{0.6} \mathrm{Ba}_{0.4} \mathrm{As}$ are the follows: the width of forbidden gap is $E g=1 \mathrm{eV}$, the lattice is constant $a=5.73 \AA$.

\section{References}

1. H. Rissel, I. Ruge, Ion implantation. Edited by Gusevoy M.I Moscow, Nauka, 1983, 360 p.

2. N. Lieske, R. Nezel. Auger electron spectroscopy and electron energy loss spectroscopy studies of the formation of silicon nitride by implanting low energy nitrogen ione into silicon //Thin Solid Films 86 (1) p. 7 - 44 (1981).

3. V.G. Lifshits. Electron spectroscopy and atomic processes on the surface of silicon. Moscow, Nauka, p. 200 (1985)
4. M.T. Normuradov, B.E. Umirzakov. Energetic spectra of the surface of solid state, implanting of low energy ions. Tashkent. Pub.:"Fan". 1989. 158 p.

5. U.A. Arifov. Interaction of the atomic particles with solid surface. Moscow, Nauka, p. 22 - 24, 242 - 243 (1968).

6. V.K. Adamchuk, S.I. Fedosenko. Investigation of the process forming the "semiconductor - metal" contact by ultraviolet photoelectron spectroscopy method. I/Izv RAN. Ser. Phys. 43 (3), p. 523 - 528 (1979).

7. Molecular beam epitaxy and heterostructures. Edited by Leroy L. Chang, Klaus Ploog. Martinus Nijhoff Publishers, $1985,520 \mathrm{p}$

8. B.E. Umirzakov, M.T. Normuradov, A.K. Tashatov, I.N Kodirov. Changes of electronic structure of Pd, Si, GaAs, $\mathrm{CaF}_{2}$ surface while low energy ion implantation and the following annealing. //9 th International Confer. on Surface Modification of Metals by Ion Beams. San Sebastian - Spain, 1995, p. 228.

9. F.G. Djurabekova, T.S. Pugacheva, D.A. Tashmukhamedova, B.E. Umirzakov. Barium concentration profiles for $\mathrm{GaAs}$ under high dose low energy $\mathrm{Ba}^{+}$ions bombing. //Proc. of the 13th International Conference "Ion-Surfaces Interactions", Moscow, 1997, 2, p.224. 